\title{
Non-oliguric renal failure with nephromegaly as the presenting manifestation of acute lymphoblastic leukemia in a young adult: An unusual case report
}

\author{
Ashwin Patil ${ }^{1}$, Rushi Deshpande ${ }^{1}$, Sudhiranjan Dash ${ }^{1}$, Ganpati Bhat ${ }^{2}$ and Shaurya Mehta ${ }^{\text {* }}$ \\ ${ }^{1}$ Department of Nephrology, Jaslok Hospital, Mumbai, India \\ ${ }^{2}$ Department of Oncology, Jaslok Hospital, Mumbai, India
}

\begin{abstract}
Renal failure as the initial presenting manifestation of acute lymphoblastic leukemia (ALL) is uncommon. In acute leukemia, commonly occurring renal complications are due to treatment-related side effects such as tumor lysis syndrome, use of nephrotoxic drugs and sepsis. Here, we are presenting an interesting case of 25 years old lady with non-oliguric acute kidney injury (AKI) of no apparent cause and vague abdominal pain. Investigations revealed anemia with mild leukocytosis, no immature cells on peripheral blood smear, deranged renal functions and bilateral uniformly enlarged non-cystic kidneys. Renal and subsequently, bone marrow biopsies revealed diagnosis of ALL. Renal failure with nephromegaly as the initial presentation of ALL is a rarity.
\end{abstract}

\section{Introduction}

In acute leukemia, renal complications are commonly due to treatment-related side effects such as tumor lysis syndrome, use of nephrotoxic drugs, sepsis, hyperuricemia and hypercalcemia $[1,2]$. Hyperuricemia is a well-recognized complication of tumor lysis syndrome [2] and in most cases, it occurs after the initiation of chemotherapy. Renal failure as the initial presenting manifestation of acute lymphoblastic leukemia (ALL) is uncommonly seen.

Here, we report a case of a young lady who presented with unexplained acute renal failure and on evaluation, was found to have ALL with predominant renal manifestations.

\section{Case report}

A 25 years old unmarried female residing in Mumbai, visited our hospital with complaints of

- Insidious onset and progressive abdominal distension since 2 months

- Generalised weakness and weight loss (not quantified) since 1 month

- vague abdominal pain off \& on since 20 days which was dull aching in nature, localised to lumbar regions.

- Swelling in both lower limbs since 10 days, pitting in nature and aggravating on prolonged standing.

This lady was recently diagnosed with Hypertension 1 month ago during a visit to a local doctor for abdominal discomfort. There was no history of oliguria, facial puffiness, breathlessness, hematuria, frothy urine and burning micturition. There was no other significant relevant history including no other co-morbidities.

On examination, significant findings included high BP (160/100 $\mathrm{mmHg}$ in right upper limb in supine position), pedal edema in both legs and bilateral enlarged, ballotable non-tender kidneys on palpation. BMI was 21.6. Rest of general and systemic examination was unremarkable.

Salient Investigations were as follows: $\mathrm{Hb} 10.5 \mathrm{gm} / \mathrm{dl}$, mildly elevated TLC 13200 cells $/ \mathrm{cmm}$, Platelet count 3.22 lacs, Normal peripheral smear.

Creatinine $5.32 \mathrm{mg} / \mathrm{dL}, \quad B U N 34 \mathrm{mg} / \mathrm{dL}, \mathrm{Na} / \mathrm{K} \quad 135 / 5.4 \mathrm{mEq} / \mathrm{L}$, Uric acid $10.4 \mathrm{mg} / \mathrm{dL}$, Calcium $9.4 \mathrm{mg} / \mathrm{dL}$, Albumin $3.3 \mathrm{~g} / \mathrm{dL}, \mathrm{LDH}$ $1104 \mathrm{U} / \mathrm{L}$, ESR $75 \mathrm{~mm} / \mathrm{hr}$.

Complete urine examination showed albuminuria, occasional RBC \& 6-8 pus cells per high power field. ANA/C3/C4/ANCA tests were within normal limits. USG abdomen showed bilateral enlarged kidneys, Right $16.6 \times 10 \mathrm{~cm}$ and Left $16.7 \times 8.4 \mathrm{~cm}$, with raised echogenicity, CMD well maintained and bilateral enlarged ovaries.

HRCT CHEST did not show any lymphadenopathy and pulmonary infiltrates.

$\mathrm{CT}$ abdomen (non-contrast) revealed following findings: (Figure 1)

- Rt kidney $17.1 \times 8.2 \mathrm{~cm}$, Lt $15.2 \times 8.2 \mathrm{~cm}$ with thickened parenchyma. Slight lobulated contours with parenchymal hypoenhancement and irregular areas of preserved normal enhancement within its parenchyma. Mild dilatation of pelvicalyceal system seen. No visible multiple parenchymal cysts or focal lesion. No visible lymphadenopathy.

- Lt ovary $4.5 \times 3.5 \mathrm{~cm}$, Rt 5.8 x $4.8 \mathrm{~cm}$. Multiple tiny follicles seen with the enlarged ovaries

${ }^{*}$ Correspondence to: Shaurya Mehta, Department of Nephrology, Jaslok Hospital, Mumbai, India, E-mail: shaurya.mehta.91@hotmail.com

Received: November 27, 2020; Accepted: December 10, 2020; Published: December 14, 2020 


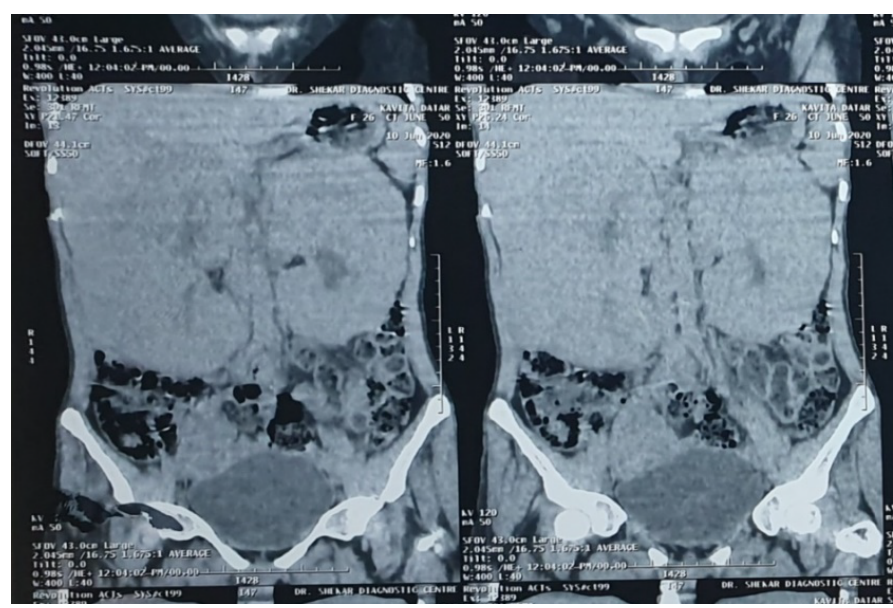

Figure 1. Bilateral massive nephromegaly on CT Abdomen

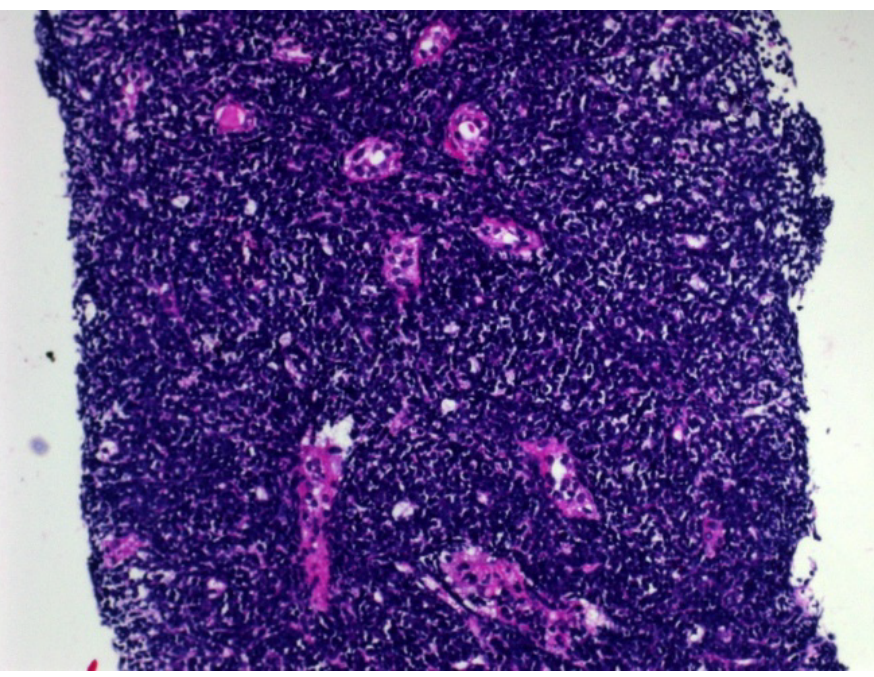

Figure 2. Renal Biopsy: Interstitium is replaced by small to medium sized lymphocytes which are present in sheets and have completely overwhelmed the parenchyma

A diagnostic kidney biopsy (Figure 2) showed

- Normal glomeruli with thin basement membrane

- Patent capillary lumina

- Only a few intact tubules showing severe hydropic changes

- Entire interstitium is replaced by small to medium sized lymphocytes which are present in sheets and have completely overwhelmed the entire parenchyma.

- Immunoperoxidase staining showed tumor cells staining positive (3+) with Tdt, PA5 and 1467

- A large number of cells stained positive (2+) with CD19, CD 79q and CD10

- IgG, IgA, C3, C1q, Kappa and Lambda were all negative.

- Above findings suggestive of B- lymphoblastic lymphoma leukemia

The patient was subjected to bone marrow biopsy \& aspiration for histopathology and immunophenotyping. Findings included Myeloid:Erythroid ratio $3: 1$ and $45 \%$ infiltration of abnormal Lymphoid cells. Flowcytometry showed positivity for CD19 (bright positive 90), CD 22 (positive 88 ), CD38 (bright positive 85), CD200 (dim positive 87 ) and CD 79a (dim positive 87 ) favouring a diagnosis of ALL. Karyotyping showed monosomy 13 and t $(1 ; 19)$.

The patient was managed as per Acute Lymphoblastic Leukemia protocols.

\section{Discussion}

Leukemia and lymphoma are hematologic malignancies derived from bone marrow or lymphoid cells, respectively and can involve other organs, including the kidneys.

Kidney manifestations encompass a broad spectrum of diseaseprerenal acute kidney injury (AKI), acute tubular necrosis (ATN), reno-vascular disease, parenchymal infiltration, obstruction, glomerulopathies, and electrolyte and acid-base abnormalities. Kidney injury may result from the underlying malignancy per se or as a secondary complication of therapy or associated infections.

Kebaili, et al. [3] described a 13 years old female with left solid renal mass discovered on routine ultrasound imaging 32 months after complete remission. Similarly, De Alivia, et al. [4] described a 16 years old male presenting with painless hematuria and solitary exophytic renal mass 36 months after completing chemotherapy for ALL. In both cases, renal biopsy confirmed lymphoblastic infiltration while bone marrow biopsy/aspirate was histologically normal.

The kidney is the most common extra-reticular and extrahematopoietic organ infiltrated by leukemia and lymphoma, with infiltration seen in $60 \%$ to $90 \%$ of patients with hematologic malignancy [5]. Kidney dysfunction varies from asymptomatic to severe and requiring renal replacement therapy. In a series of 1200 autopsy cases, the prevalence of kidney infiltration was $63 \%$ in chronic lymphocytic leukemia (CLL), $54 \%$ in acute lymphoblastic leukemia (ALL), 34\% in chronic myeloid leukemia (CML), and 33\% in acute myeloid leukemia (AML) [6].

Kidney failure as a primary manifestation due to lymphomatous or leukemic infiltration is rare especially in a young adult. AKI from infiltration is seen in only $1 \%$ of cases of all patients with acute leukemias [7]. Symptoms and signs associated with infiltration include flank pain, hematuria, abdominal distension, or hypertension [7]. Infiltration has to be bilateral in nature, and enlargement is usually uniform. Kidney biopsy is often useful in these patients for two reasons. First, the extent and location of infiltration tends to influence prognosis. Interstitial infiltration tends to be associated with AKI, as opposed to glomerular infiltration, which presents as a glomerulopathy. Secondly, biopsy may help differentiate subtypes of lymphoma or leukemia and alter disease treatment. Specific stains can be used to identify cellular markers. Targeting these cells with chemotherapy is important because improvement of kidney function depends on treatment of the underlying disease. Regression and improvement in kidney function should be prompt (i.e, occurring sometime within 2-3 days of therapy). Kidney failure resulting from infiltration is thought to be secondary to acute tubular compression and disruption of the kidney microvasculature from increased interstitial pressure leading to ATN.

Our patient was started on alkaline diuresis, dexamethasone and uric acid reducing agents. Potassium homeostasis was maintained medically. Serial biochemistry tests were done as depicted in Figure 3.

Our case is unique in that non-oliguric renal failure was the presenting feature with nearly normal peripheral blood counts and smear, hyperuricemia, no evidence of lymphadenopathy, bilateral enlarged kidney, elevated $\mathrm{LDH}$, and evidence of lymphoblastic leukemia on initial kidney and then, bone marrow biopsies with immmunohistochemistry analysis. 


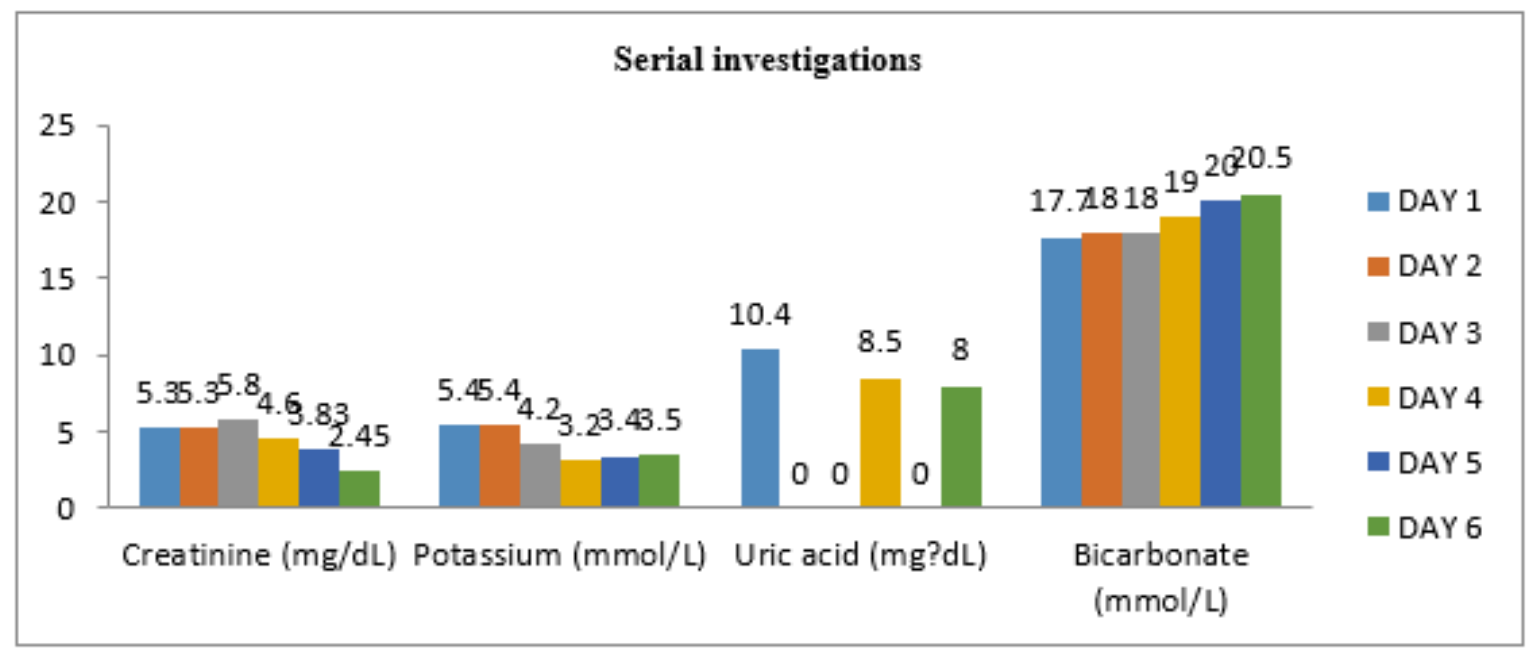

*Days when investigation was not done is represented by " 0 "

Figure 3. Serial biochemistry tests

A clinical lesson to be learnt in this case is that one has to search for a cause for unexplained AKI. Before labelling a patient as AKI of unknown etiology, it is prudent to evaluate the patient thoroughly. In this case the clue for underlying disease came from presence of hyperuricemia, deranged renal functions, high $\mathrm{LDH}$ and bilateral uniformly enlarged non-cystic kidneys.

\section{Acknowledgement}

We would like to thank Dr. Shaila Khubchandani, Consultant, Department of Pathology, Jaslok Hospital, Mumbai, India, for her expert opinion on kidney and bone marrow biopsies.

\section{Funding}

None.

\section{Conflict of interests}

The authors declare no competing interests.

\section{References}

1. Munker R, Hill U, Jehn U, Kolb HJ, Schalhorn A (1998) Renal complications in acute leukemias. Haematologica 83: 416-421. [Crossref]

2. Lommatzsch SE, Bellizzi AM, Cathro HP, Rosner MH (2006) Acute renal failure caused by renal infiltration by hematolymphoid malignancy. Ann Diagn Pathol 10 230-234. [Crossref]

3. Kebaili K, Manel AM, Chapelon C, Taylor P, Philippe N, et al. (2000) Renal enlargement as presentation of isolated renal relapse in childhood leukemia. J Pediatr Hematol Oncol 22: 454-456.

4. Alivia De, Menell J (2010) Isolated Renal Relapse in ALL. J Pediatr Hematol Oncol 32: 150-151. [Crossref]

5. Richmond J, Sherman RS, Diamond HD, Craver LF (1962) Renal lesions associated with malignant lymphomas. Am J Med 32: 184-207. [Crossref]

6. Barcos M, Lane W, Gomez GA, Freeman A, Preisler H, et al. (1987) An autopsy study of 1206 acute and chronic leukemias (1958 to 1982). Cancer 60: 827-837. [Crossref]

7. Lundberg WB, Cadman ED, Finch SC, Capizzi RL (1977) Renal failure secondary to leukemic infiltration of the kidneys. Am J Med 62: 636-642. [Crossref]

Copyright: (C2020 Patil A. This is an open-access article distributed under the terms of the Creative Commons Attribution License, which permits unrestricted use, distribution, and reproduction in any medium, provided the original author and source are credited. 